\title{
CESÀRO AND BOREL-TYPE SUMMABILITY
}

\author{
DAVID BORWEIN AND TOM MARKOVICH
}

(Communicated by R. Daniel Mauldin)

\begin{abstract}
Though summability of a series by the Cesàro method $C_{p}$ does not in general imply its summability by the Borel-type method $(B, \alpha, \beta)$, it is shown that the implication holds under an additional condition.
\end{abstract}

1. Introduction. Suppose throughout that $\sum_{n=0}^{\infty} a_{n}$ is a series with partial sums $s_{n}:=\sum_{k=0}^{n} a_{k}$, and that $\alpha>0$ and $\alpha N+\beta>0$ where $N$ is a nonnegative integer. The series $\sum_{n=0}^{\infty} a_{n}$ is said to be summable $(B, \alpha, \beta)$ to $s$ if

$$
\alpha e^{-x} \sum_{n=N}^{\infty} s_{n} \frac{x^{\alpha n+\beta-1}}{\Gamma(\alpha n+\beta)} \rightarrow s \quad \text { as } x \rightarrow \infty .
$$

The Borel-type summability method $(B, \alpha, \beta)$ is regular, and $(B, 1,1)$ with $N=0$ is the standard Borel summability method $B$.

We shall also be concerned with the Cesàro summability method $C_{p}(p>-1)$ and the Valiron method $V_{\alpha}$ defined as follows:

where

$$
\sum_{n=0}^{\infty} a_{n}=s\left(C_{p}\right) \quad \text { if } c_{n}^{p}:=\frac{s_{n}^{p}}{\left(\begin{array}{c}
n+p \\
n
\end{array}\right)} \rightarrow s \quad \text { as } n \rightarrow \infty
$$

$$
\begin{gathered}
s_{n}^{p}:=\sum_{k=0}^{n}\left(\begin{array}{c}
n-k+p-1 \\
n-k
\end{array}\right) s_{k} \\
\sum_{n=0}^{\infty} a_{n}=s\left(V_{\alpha}\right) \quad \text { if }\left(\frac{\alpha}{2 \pi n}\right)^{1 / 2} \sum_{k=0}^{\infty} \exp \left(-\frac{\alpha(n-k)^{2}}{2 n}\right) s_{k} \rightarrow s \quad \text { as } n \rightarrow \infty
\end{gathered}
$$

Consider the series $\sum_{n=1}^{\infty} a_{n}:=\sum_{n=1}^{\infty} n^{a-1} \exp \left(A i n^{a}\right)$ where $A>0$ and $0<$ $a<1 / 2$. It is known [5, p. 213] that this series is summable $C_{p}$ for every $p>0$ but is not convergent. However, since $a_{n}=o\left(n^{-1 / 2}\right)$, it follows by the Borwein Tauberian Theorem [1, Theorem 1] that the series is not summable $(B, \alpha, \beta)$ for any $\alpha$ and $\beta$. This example shows that, in general, summability $C_{p}$ does not imply summability $(B, \alpha, \beta)$. The following theorem indicates how to strengthen the $C_{p}$ summability hypothesis in order to ensure summability $(B, \alpha, \beta)$.

THEOREM 1. Suppose that $p$ is a nonnegative integer and that $c_{n}^{p}=s+o\left(n^{-p / 2}\right)$ as $n \rightarrow \infty$. Then $\sum_{n=0}^{\infty} a_{n}=s(B, \alpha, \beta)$.

The special case $\alpha=\beta=1, p=1$ of Theorem 1 has been proved by Hardy [5, Theorem 149]. Hardy and Littlewood [4, §3] proved that the condition

Received by the editors July 14, 1987.

1980 Mathematics Subject Classification (1985 Revision). Primary 40G05, 40G10.

This research was supported in part by the Natural Sciences and Engineering Council of Canada. 
$c_{n}^{p}=s+o\left(n^{-1 / 2}\right)$ is not sufficient for the summability of $\sum a_{n}$ by the Borel method. Hyslop [7, Theorem VIII] has obtained a more general result than Hardy, namely the case $\alpha=\beta=1$ of Theorem 1. More recently, Swaminathan [10] has proved Theorem 1 with $p=1$ and $(B, \alpha, \beta)$ summability replaced by the more general $F(a, q)$ summability introduced by Meir [9].

\section{Preliminary results.}

LEMMA 1 [8, LEMMA 7]. Let $m<x_{0}<n-1$ where $m, n$ are integers and let the nonnegative function $f(x)$ be increasing on $\left[m, x_{0}\right]$ and decreasing on $\left[x_{0}, n\right]$. Then

$$
\sum_{k=m}^{n} f(k) \leq \int_{m}^{n} f(x) d x+f\left(x_{0}\right)
$$

LEMMA 2 [2, THEOREM 3]. Suppose that $s_{n}=O\left(n^{r}\right)$ where $r \geq 0$. Then $\sum_{n=0}^{\infty} a_{n}=s(B, \alpha, \beta)$ if and only if $\sum_{n=0}^{\infty} a_{n}=s\left(V_{\alpha}\right)$.

THEOREM 2 (CF. [6, THEOREM 2]). Suppose that $p$ is a nonnegative integer and that $c_{n}^{p}=s+o\left(n^{-p / 2}\right)$ as $n \rightarrow \infty$. Then $\sum_{n=0}^{\infty} a_{n}=s\left(V_{\alpha}\right)$.

PROOF. Suppose, as we may without loss of generality, that $s=0$.

Let $v_{n}(x):=\exp \left(-\alpha(n-x)^{2} / 2 n\right)$ and denote the $p$ th difference of $v_{n}(k)$ by $\Delta^{p} v_{n}(k)$, so that

$$
\Delta^{p} v_{n}(k)=\sum_{r=0}^{p}\left(\begin{array}{l}
p \\
r
\end{array}\right)(-1)^{r} v_{n}(k+r) .
$$

Applying Abel's partial summation formula $p(<m)$ times, we have that

$$
\sum_{k=0}^{m} s_{k} v_{n}(k)=\sum_{k=0}^{m-p} s_{k}^{p} \Delta^{p} v_{n}(k)+\sum_{r=0}^{p-1} s_{m-r}^{r+1} \Delta^{r} v_{n}(m-r) .
$$

Letting $m \rightarrow \infty$ and applying the limitation theorem for Cesàro summability [5, Theorem 46], we see that

$$
F(n):=\sum_{k=0}^{\infty} s_{k} v_{n}(k)=\sum_{k=0}^{\infty} s_{k}^{p} \Delta^{p} v_{n}(k) .
$$

In order to prove the theorem we must show that $F(n)=o\left(n^{1 / 2}\right)$. Since, by the hypothesis, $s_{k}^{p}=o\left(k^{p / 2}\right)$ as $k \rightarrow \infty$ and $k^{p / 2} \Delta^{p} v_{n}(k)=o\left(n^{1 / 2}\right)$ as $n \rightarrow \infty$, it suffices to show that

$$
G(n):=n^{-1 / 2} \sum_{k=0}^{\infty} k^{p / 2}\left|\Delta^{p} v_{n}(k)\right|
$$

is bounded.

It is familiar that $\Delta^{p} v_{n}(k)=(-1)^{p} v_{n}^{(p)}(k+c)$ for some $c \in[0, p]$. Hence there is a $\theta=\theta(n, k) \in[0, p]$ such that

$$
\left|\Delta^{p} v_{n}(k)\right| \leq\left|v_{n}^{(p)}(k+\theta)\right|
$$


Since $v_{n}^{(p)}(x)=v_{n}(x) \sum_{0 \leq r \leq p / 2} b_{r}(n-x)^{p-2 r} n^{r-p}$, where the $b_{r}$ 's are constants, we get from (1) and (2) that

$$
G(n)=O\left(\sum_{0 \leq r \leq p / 2}\left|b_{r}\right| n^{r-p-1 / 2} \sum_{k=0}^{\infty} k^{p / 2}|n-k-\theta|^{p-2 r} v_{n}(k+\theta)\right) .
$$

Therefore to establish that $G(n)$ is bounded it is enough to show that, for $0 \leq r \leq$ $p / 2$ and $0 \leq \theta \leq p$,

$$
H(n):=\sum_{k=0}^{\infty} k^{p / 2}|n-k-\theta|^{p-2 r} v_{n}(k+\theta)=O\left(n^{p-r+1 / 2}\right) .
$$

Write

$$
\begin{aligned}
H(n) & =\left\{\sum_{k=0}^{n-p-1}+\sum_{k=n-p}^{n}+\sum_{k=n+1}^{\infty}\right\} k^{p / 2}|n-k-\theta|^{p-2 r} v_{n}(k+\theta) \\
& :=\sum_{1}+\sum_{2}+\sum_{3} .
\end{aligned}
$$

Since $|n-k-\theta| \leq 2 p$ for $0 \leq \theta \leq p$ and $n-p \leq k \leq n$, and $0<v_{n}(k+\theta) \leq 1$, it is immediate that

$$
\sum_{2}=O\left(n^{p / 2}\right)
$$

Next, setting $f(x):=x^{p-2 r} \exp \left(-\alpha x^{2} / 2 n\right)$ and applying Lemma 1 , we have that

$$
\begin{aligned}
\sum_{1} & \leq \sum_{k=0}^{n-p-1} k^{p / 2}(n-k)^{p-2 r} v_{n}(k+p) \leq \sum_{k=p}^{n-1} k^{p / 2}(n-k+p)^{p-2 r} v_{n}(k) \\
& \leq M n^{p / 2} \sum_{k=p}^{n-1} f(n-k) \leq M n^{p / 2} \sum_{k=1}^{n} f(k) \\
& \leq M n^{p / 2} \int_{1}^{n} f(x) d x+M C n^{p / 2}\left(\frac{(p-2 r) n}{\alpha}\right)^{p / 2-r}
\end{aligned}
$$

where $M:=(1+p)^{p-2 r}$ and $C:=\exp (r-p / 2)$. Letting $u=\alpha x^{2} / 2 n$, we get that

$$
\sum_{1}=O\left(n^{p-r+1 / 2} \int_{0}^{\infty} u^{(p-1) / 2-r} e^{-u} d u\right)+O\left(n^{p-r}\right)=O\left(n^{p-r+1 / 2}\right) .
$$

Further, with $M$ and $f(x)$ as above and $g(x):=x^{3 p / 2-2 r} \exp \left(-\alpha x^{2} / 2 n\right)$, we see that

$$
\begin{aligned}
\sum_{3} & \leq \sum_{k=n+1}^{\infty} k^{p / 2}(k-n+p)^{p-2 r} v_{n}(k) \\
& \leq M\left(\sum_{k=n+1}^{2 n}+\sum_{k=2 n+1}^{\infty}\right) k^{p / 2}(k-n)^{p-2 r} v_{n}(k) \\
& \leq M(2 n)^{p / 2} \sum_{k=1}^{n} f(k)+M 2^{p / 2} \sum_{k=n+1}^{\infty} g(k):=\sum_{3,1}+\sum_{3,2} .
\end{aligned}
$$


As above $\sum_{3,1}=O\left(n^{p-r+1 / 2}\right)$. And finally, as $n \rightarrow \infty$,

$$
\begin{aligned}
\sum_{3,2} & =O\left(\int_{n}^{\infty} g(x) d x\right)+o(1) \\
& =O\left(n^{3 p / 4-r+1 / 2} \int_{\alpha n / 2}^{\infty} u^{3 p / 4-r-1 / 2} e^{-u} d u\right)+o(1) \\
& =o(1) .
\end{aligned}
$$

Thus,

$$
\sum_{3}=O\left(n^{p-r+1 / 2}\right)+o(1) \quad \text { as } n \rightarrow \infty .
$$

It now follows from (3)-(6) that $H(n)=O\left(n^{p-r+1 / 2}\right)$. This completes the proof.

3. Proof of Theorem 1. The limitation theorem for Cesàro summability [5, Theorem 46] implies that $s_{n}=o\left(n^{p}\right)$. Therefore, by Theorem 2 and Lemma 2, we have that $\sum_{n=0}^{\infty} a_{n}=s(B, \alpha, \beta)$.

4. Related results. The methods of Euler $E_{\delta}$, Meyer-Konïg $S_{\delta}$, and Taylor $T_{\delta}(0<\delta<1)$ are defined as follows:

$$
\begin{gathered}
\sum_{n=0}^{\infty} a_{n}=s\left(E_{\delta}\right) \quad \text { if } \quad \sum_{k=0}^{n}\left(\begin{array}{c}
n \\
k
\end{array}\right) \delta^{k}(1-\delta)^{n-k} s_{k} \rightarrow s \quad \text { as } n \rightarrow \infty \\
\sum_{n=0}^{\infty} a_{n}=s\left(S_{\delta}\right) \quad \text { if }(1-\delta)^{n+1} \sum_{k=0}^{\infty}\left(\begin{array}{c}
n+k \\
k
\end{array}\right) \delta^{k} s_{k} \rightarrow s \quad \text { as } n \rightarrow \infty \\
\sum_{n=0}^{\infty} a_{n}=s\left(T_{\delta}\right) \quad \text { if }(1-\delta)^{n+1} \sum_{k=0}^{\infty}\left(\begin{array}{c}
n+k \\
k
\end{array}\right) \delta^{k} s_{n+k} \rightarrow s \quad \text { as } n \rightarrow \infty
\end{gathered}
$$

These methods, as well as the Borel-type and Valiron methods, are contained in the $F(a, q)$ family of methods mentioned in the introduction. The following theorem generalizes Swaminathan's result [10], via Theorem 2 and [3, Satz III], for the Euler, Meyer-Konïg, and Taylor methods.

THEOREM 3. Suppose that $p$ is a nonnegative integer and that $c_{n}^{p}=s+o\left(n^{-p / 2}\right)$ as $n \rightarrow \infty$. Then for $0<\delta<1$, the series $\sum_{n=0}^{\infty} a_{n}$ is summable to $s$ by the $E_{\delta}$, $S_{\delta}$, and $T_{\delta}$ methods.

\section{REFERENCES}

1. D. Borwein, A Tauberian theorem for Borel-type methods of summability, Canad. J. Math. 21 (1969), 740-747.

2. D. Borwein and T. Markovich, A Tauberian theorem concerning Borel-type and Cesàro methods of summability, (submitted).

3. G. Faulhaber, Äquivalenzsätze für die Kreisverfahren der Limitierungstheorie, Math. Z. 66 (1956), 34-52.

4. G. H. Hardy and J. E. Littlewood, Theorems concerning the summability of series by Borel's exponential method, Rend. Circ. Mat. Palermo 41 (1916), 36-53.

5. G. H. Hardy, Divergent series, Oxford, 1949. 
6. J. M. Hyslop, On the summability of series by a method of Valiron, Proc. Edinburgh Math. Soc. (2) 4 (1936), 218-223.

7. _ The generalisation of a theorem on Borel summability, Proc. London Math. Soc. (2) 41 (1936), 243-256.

8. B. Kwee, An improvement on a theorem of Hardy and Littlewood, J. London Math. Soc. (2) 28 (1983), 93-102.

9. A. Meir, Tauberian constants for a family of transformations, Ann. of Math. (2) 78 (1963), 594-599.

10. V. Swaminathan, $A$ note on the family $F(a, q)$ of summability methods, Math. Z. 138 (1974), 119-122.

Department of MAThematics, UniVERsity of Western ONTARIO, LONDON, ONTARIO, CANADA N6A 5B7 